\title{
Exploring the Dependency of Type of School and Age with Adolescent Connectedness
}

\author{
Prof. Salomé Schulze \\ Dr. Narainsamy Naidu \\ University of South Africa, South Africa \\ E-mail: schuls@unisa.ac.za
}

\author{
Doi:10.5901/mjss.2014.v5n2p323
}

\begin{abstract}
The research aimed to explore the connectedness of South African adolescents and identify significant dependencies thereof with type of school and age (grade level). To this end, 486 Grade 8 and 330 Grade 11 students enrolled at four diverse schools completed the Hemingway Measure of Adolescent Connectedness (MAC) questionnaire. The questionnaire gauged student connectedness on 15 subscales. The students were best connected to the future, religion and themselves in the present. Regarding family, in rank order they were connected to their mothers, their siblings and lastly to their fathers. They were least connected to reading, their neighbourhoods and to romantic partners. Statistically significant differences were found between the connectedness of the students of the four schools, and of the two grade levels. In general, the students of school $A$, which served a high socio-economic area, were best connected. The Grade 8 students were also better connected than the Grade 11 students on several of the subscales. Some recommendations for improving adolescent connectedness and for future research were made.
\end{abstract}

Keywords: Adolescent connectedness, MAC questionnaire, South Africa, school effect, age effect

\section{Introduction}

Researchers of adolescent connectedness are confronted with an array of terminology that seems to vary from author to author but is related to the issue of 'connectedness' or 'autonomy'. Autonomy is often referred to as 'agency' or 'separation-individuation' (Kagitcibasi 2005, p. 403). Connectedness (Karcher, 2005), on the other hand, is associated with 'relatedness' (Kagitcibasi, 2005), 'belongingness beliefs' (Allen \& Bowles, 2012; Nichols, 2008), 'engagement' (e.g., Gallant, Smale \& Arai, 2010), 'a sense of membership' (Portwood, Ayers, Kinnison, Waris \& Wise, 2005), 'closeness' (Smetana, 2011) and 'attachment' (Bosma \& Gerslma, 2003, p. 450). The terms used suggest that connectedness is the degree to which individuals experience the people and places in their lives as personally meaningful and important.

Connectedness has frequently been portrayed as conflicting with a sense of autonomy. Autonomy is at the core of the Social Cognitive theory of Bandura (1989) and has, together with agency, been portrayed as a positive psychological trait (Seiffge-Krenke \& Pakalniskiene, 2011). However, connectedness, which differs from neediness ('anxious concerns regarding possible rejection') (Rude \& Burham, 1995, p. 337), is also a strong human need, as argued by Bowlby (1988). Moreover, connectedness is associated with a range of psychological benefits, such as overall physical health and psychological well-being that include a positive self-esteem, self-efficacy, and life satisfaction (Haslam, Jetten, Postmes \& Haslam, 2009); as well as happiness (Sharma \& Malhotra, 2010). In contrast, a lack of social connectedness and support are related to loneliness and adjustment difficulties (Duru, 2008); self-alienation, a lack of meaning and purpose in life; and poor health (Bellingham and co-authors in Townsend \& McWhirter, 2005, p. 192).

Considering the above and in the light of the amount of time adolescents spend in school, schools are important to build social networks for adolescents and to enhance their feelings of connectedness, as also argued by Allen and Bowles (2012, p. 108). Therefore, more information is needed on the influence of the type of school that is attended by students of different age groups on their feelings of connectedness (Allen \& Bowles, 2012, p. 109; Nichols, 2008, p. 146). This may shed light on the kind of interventions or modifications of school structures that are needed to enhance the connectedness of the students.

A South African case study investigated qualitatively factors that impeded school connectedness in one school in the province of KwaZulu-Natal (Rawatlal \& Petersen, 2012.) The following factors were identified: non-relevant education policies, top-down management practices and ineffective discipline strategies at the school management level; negative 
peer influence on the interpersonal level; emotional incompetence as well as a lack of self-regulation skills or of a future orientation on the intra-personal level. The absence of a future focus was attributed to poor discipline and a lack of warmth at home, to the school's heavy focus on assessment and to limited post-school opportunities in South Africa. The case study prompted this research which explores adolescent connectedness in a different province of the country (Gauteng), using a quantitative approach to enable generalisation. In particular, the aim is to explore the adolescent connectedness of students of two grade levels at four diverse school types.

The remainder of the article explains the conceptual framework, the research design, and the results of the study. The article ends with the most significant conclusions and recommendations.

\section{Conceptual Framework}

Bronfenbrenner's Ecological system's Theory $(1979,1999)$ clarifies how the family is the first system to which a child belongs, and thereafter the school and the community. The development of the child's feelings of connectedness is influenced by the quality of the environment at each of multiple levels, namely the microsystem, mesosystem, exosystem and macrosystem. When a child is at school, the microsystem is the social networks of family, friends, teachers and peers; the mesosystem is the school management and teaching processes that determine the school culture; the exosystem is the broader community and the macrosystem refers to policies and laws.

Complementing the above is Self-determination Theory (SDT). SDT indicates that autonomy and connectedness (together with competence) are basic human needs and the satisfaction of all these needs is required for healthy psychological functioning, as mentioned (Chirkov, Kim, Ryan \& Kaplan, 2003; Ryan \& Deci, 2000). Ryan and Deci (2000, p. 12) maintain that by failing to provide support for autonomy and relatedness of children, socialising agents and organisations contribute to alienation and ill-being.

According to Kagitcibasi (2005), autonomy and agency overlap in meaning in the sense that autonomy refers to being an agent and to act willingly. Similarly, separateness and relatedness/connectedness can be both agentic and volitional. Autonomy has two dimensions: (i) interpersonal distance, i.e. separateness versus connectedness and (ii) agency, i.e. autonomy (subject to own rules) or heteronomy (subject to another's rule). An individual's standing on the first dimension (interpersonal distance) does not imply any particular standing on the second dimension (agency). In other words, an individual can be high on connectedness and on autonomy as also acknowledged by SDT, although some cultures value the one more than the other. For example, Eastern cultures value connectedness more than Western cultures (Dwairy \& Achoui, 2010). Yu (2011) determined that In the Korean culture, the maintenance of adolescents' connectedness to their parents undermined their' attainment of autonomy although this may not be the case in other cultures.

During adolescence, autonomy and connectedness assume special significance. For example, regarding adolescents' connectedness with their parents, some authors view distancing from the parents as a healthy development (autonomy as separation-individuation). Others (e.g. Kagitcibasi, 2005), who define autonomy as agency argue that close relationships with parents are associated with adolescent well-being. This leads to the concept of the autonomousrelated self. Fathers (rather than mothers), seem to be models for a balance between separateness and connectedness (Shulman \& Klein, 1993).

Depending on family interaction patterns, different kinds of selves can develop: (i) the heteronomous-related self (high in connectedness and low in autonomy) develops in a family model of total interdependence; (ii) the autonomousseparate self (high in autonomy and low in connectedness) develops in the family model of independence and selfreliance; (iii) the autonomous-related self (high in both connectedness and autonomy) develops in the family model of psychological interdependence with autonomy; and (iv) the separated but heteronomous self develops in a family with neglecting and rejecting parents.

The above mentioned model focuses on adolescent, parent connectedness but can also be applicable to connectedness to others such as siblings, peers or friends. The adolescent cannot be placed into any neat category of 'connectedness' since there are degrees of variation along the two dimensions of agency and interpersonal distance. Moreover, the relative salience and the avenues for satisfaction of the need for connectedness/autonomy change across the life span. Their modes of expression are also determined by culture as one of the factors related to connectedness (Ryan \& Deci, 2000). 


\section{Factors Related to Adolescent Connectedness}

Libbey (2004, p. 274) points out that the literature offers a range of terms related to school connectedness such as school bonding, attachment, belonging, engagement, school climate or notions of territory (Allen \& Bowles, 2012; Loukas, Ripperger-Suhler \& Horton, 2009, p. 804). Notwithstanding the particular concept used, school connectedness is an important protective factor against substance abuse, school absenteeism, early sexual initiation, and violence and risky behaviour (Davis-Alldritt, 2012). School connectedness fosters self-esteem and self-identity (Nutbrown \& Clough, 2009), and improves academic outcomes because of enhanced motivation and low absenteeism (Sánchez, Colón \& Esparza 2005). An Australian study investigated five factors (social competence, life satisfaction, trust of others and in authorities, and civic responsibilities) in a longitudinal study with adolescents. School connectedness at high school significantly predicted early adult well-being across all five factors as found by O'Connor and co-workers (in Allen \& Bowles, 2012, p.111).

Loukas et al (2009:804) draw attention to a number of theories that enhance insight into the relationship between school connectedness and early adolescent adjustment. For example, Social Control theory states that adolescents who are connected to school show less externalising problems since they strive to meet the community's expectations of them. They form supportive relationships with teachers and pro-social peers and are therefore less likely to develop emotional problems. Transactional models of development also illustrate that early adolescent adjustment contributes to school connectedness and vice versa (Sameroff 2000).

School connectedness seems to be particularly important during early adolescence. During this time, the need for interpersonal affiliation with peers and with adults who are not family increases (Buhrmester, 1990). Young adolescents who are well connected to their schools experience fewer problems. For example, $6^{\text {th }}$ and $7^{\text {th }}$ grade adolescents' levels of school connectedness were significantly related to less behaviour problems (Loukas et al, 2009, p. 808-809) as well as psychological problems later (Shochet, Dadds, Ham \& Montague, 2006).

According to Loukas et al (2009:805), in particular for the 10 to 14 year age group, feelings of school connectedness could diminish because of new developmental needs for this age group that are not always met by the school environments. While attachment to parents diminishes during this time, the need for greater connectedness to peers and other adults increases. However, in comparison to primary schools, schools during this time are more formal and impersonal, thus impeding school connectedness.

School connectedness is strengthened if the students' expectations of a school are met (Nichols, 2008, p. 165). A key factor seems to be the students' relationships with their teachers (Libbey, 2004, p.281). Students are particularly influenced by whether the teachers respect their opinions, understand their feelings, and are emotionally and academically supportive. The adolescents' connectedness to school is also enhanced by a positive school culture (fair school rules and feeling safe at school), and by social interactions, such as fitting in with others in the school setting and satisfying school friendships. If the students lack friends, their teachers' attention and care could compensate for this, and vice versa (Nichols, 2008, p. 164). If students experience relational victimisation at school, teachers' emotional support could also buffer the effects thereof (Yeung \& Leudbeater, 2010).

The kind of school that adolescents attend matters and influences the students' connectedness. In this regard, the gender composition of a school is influential, although this effect is moderated by the student's age. One study found that during early adolescence, girls in single-sex schools experienced lower levels of stress than their coeducational counterparts and that this could be attributed to their school connectedness (Brutsaert \& Van Houtte, 2004, p.58). Another factor that may be influential is whether the school offers mentoring programmes. If students participated in satisfactory mentoring programmes with other students, this improved their school connectedness and their attitudes toward themselves, adults, and the future (Portwood et al., 2005, p. 140). Karcher (2009, p. 292) confirmed that if high school students served as mentors to younger children, this facilitated improved academic self-esteem and connectedness of the mentors. Similarly, the mentees' connectedness to school and peers as well as their social skills improved (Karcher, 2005).

Even though connectedness to schools is important during adolescence, as argued, family connectedness remains crucial. Davis-Alldritt (2012) states that connectedness to family is more important than connectedness to school to prevent emotional distress, eating disorders, and suicide ideation during adolescence. Connectedness to the family seems to be stronger in families with a higher socio-economic level and where parents are more educated as determined in nine western and eastern countries (Dwairy \& Achoui, 2010). In all the cultures, the adolescents showed connectedness in rank order to their mothers, their siblings and thereafter to their fathers.

New research on adolescent-family relationships confirms that adolescents do not detach fully from parents as 
earlier researcher have claimed (Smetana, 2011, p. 23). Although adolescents feel less emotionally close to their parents and grandparents as they get older, the parents remain a source of support even though bickering may increase over minor issues related to, for example, schoolwork, friends and personal appearance. As adolescents partially separate from parents, they get closer to their friends and peers and later in adolescence, to romantic partners. To form an autonomous identity in adolescence, parents need to exhibit characteristics that facilitate psychological separateness as well as promote connectedness (Sim, 2003, p.385). Being sensitive to the adolescents' needs and being supportive are important for parent-adolescent connectedness during identity formation. Such connectedness is negatively associated with a depressed mood although religiosity also plays a role (Houltberg, Henry, Merten \& Robinson, 2011).

Religiosity provides feelings of belonging that counter feelings of worthlessness and hopelessness. A connection to God may also counter negative feelings of self and the ability to recognise connections in the family. Adolescents need to establish a personal stance on existential issues, which include religious questions, as part of the process of forming a stable identity. A study with 1,326 South African adolescents found that the students expressed a particularly strong identification with religion and the students attended church at least twice per month (Steyn, Badenhorst \& Kamper, 2010). The way that the adolescents deal with religion is related to social-political attitudes. For example, individuals who deal with religion in a dogmatic, literal way seem to be prone to racial and other forms of prejudice and cultural conservatism (Duriez, 2003, 2004). Other studies have investigated the relationship between connectedness to religion and adolescent risk behaviours. One such a study with 11 to 18 year old adolescents determined that they viewed religion as important, actively participated in religious worship and pursuits and that this was associated with a positive life orientation and decreased risk behaviours (Sinha, Cnaan \& Gelles, 2007).

Regarding adolescents' connectedness to their neighbourhoods, such linkages are elements of a complex ecological system to support the adolescent's development and learning, as Bronfenbrenner's (1979) theory has illustrated. Neighbourhoods are defined by elements such as population characteristics, social class, housing quality, urban form, opportunities for community centre involvement, the occurrence of crime and violence, and the location of private schools. If the adolescents attend schools in their own neighbourhoods, social cohesion is strengthened by the 'educational talk' of parents who are also neighbours and who send their children to the same school (Johnson, 2012). Such cohesion can influence student connectedness and learning.

With regard to reading, there is a worldwide trend for teenagers to read less (Machet, 2002, p. 57). In a South African study with adolescents from five English-medium secondary schools in Gauteng, Machet (2002) found that the students' reading patterns were influenced by, among others, their age, gender, the visual elements of the book (e.g. illustrations and the cover), the language in which it is written (English versus their home language), the particular author, whether it is connected to a television series and the book's genre (e.g. South African adolescents do not enjoy horror stories as much as their American counterparts since crime is too much of a reality for them). The girls in particular often preferred magazines over books.

Another aspect of connectedness that is important is connectedness to the future. A South African study that investigated this issue with adolescents from all socio-economic backgrounds, found that they were generally optimistic about the future, in addition to reflecting positive self-images and happiness (Steyn et al., 2010). The students aimed for successful careers and financial security.

In consideration of the foregoing exposition, the connectedness of a group of adolescents was explored. In particular, the dependency of connectedness with type of school and school grade (age) was investigated. Thus, two hypotheses were stated: There are significant differences between (i) different schools and (ii) grade levels on general connectedness and on subscales of connectedness (related to the self, others, and society). The research design and data collection methods used are explained in the next section.

\section{Research Design and Data Collection}

The sample was selected through a combination of purposeful and convenience sampling (McMillan \& Schumacher, 2010:137) and included 486 Grade 8 and 330 Grade 11 students (and were thus of different ages), from four diverse schools in a South African city. School A was a big secondary school (more than a thousand students) in an affluent area, with effective discipline and an excellent academic record as indicated by Grade 12 results. The medium of instruction was Afrikaans. School B was also a big secondary school, multi-cultural, experienced disciplinary problems and did not have a very good academic record. School C was a very small, private Afrikaans-medium Christian secondary school of a specific denomination. Finally, School D was a multi-cultural, English-medium art secondary school that specialised in the performing and the creative arts. 
Ethical clearance was obtained as well as consent from the parents and the students. The data were collected by means of (anonymously) completed class-administered questionnaires during one school period, The Hemmingway: Measure of Adolescent Connectedness (MAC) (Karcher, 2000). After obtaining permission from the author of the questionnaire, it was translated into Afrikaans for use in two of the schools. It was first pilot tested and then finalised without the need to make any changes. The MAC is made up of 72 items designed to measure the adolescents' degree of connectedness in 15 instances. These subscales include connectedness to others (e.g. parents and siblings); to the community (e.g. the neighbourhood) and to the self (e.g. self in the future). Responses to each of the subscale items implemented a five-point, Likert-type scale namely (1) "not true at all", (2) "not really true", (3) "sort of true", (4) "true", to (5) "very true". Each subscale had one reverse scored item. The items in each of the subscales reflected connecting through active involvement and through caring (e.g. "I work hard at school" and "Doing well in school is important to me.")

Data analysis was through the comparison of means and ANOVAs to test the above mentioned two hypotheses. The Cronbach's alphas on 11 subscales were between .704 and .888 except in four instances (self-in-the-present, peers, teachers and the future) where the alphas were just below .7. However, it should be emphasised that this was only an exploratory study (McMillan \& Schumacher, 2010, p.:182). Face validity was judged favourably by an expert.

\section{Results}

The means for type of school appear in Figure 1.

Figure 1: Means of four different schools

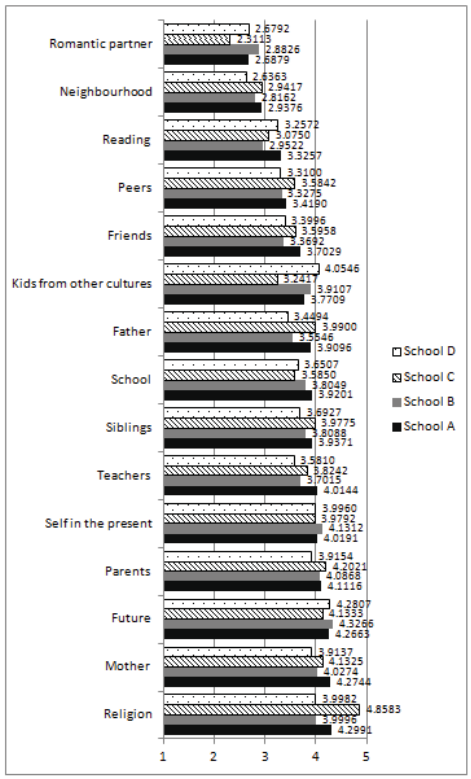

In particular, Figure 1 reveals the following:

- Students of all the schools were well connected to the future $(M>4)$;

- The highest connection for students of schools $A$ and $C$ was to religion ( $M=4.2991$ to 4.8583$)$ - for students of schools $B$ and $D$ connection to religion was also high ( $M>3.9)$;

- Adolescents at schools A, B and C were well connected to their parents, particularly to their mothers $(M>4)$, while these connections for students of school $D$ were also relatively high ( $M=3.9154$ and 3.9137 respectively); in contrast, the adolescents of school $\mathrm{D}$ were the only ones that were very well connected to kids of other cultures $(M>4)$;

- Only the adolescents of school A were highly connected to their teachers $(M=4.0144)$;

- The lowest levels of connectedness in all instances were with neigbourhoods and romantic partners $(M<3)$, and with reading $(M=2.9522$ to 3.3275$)$. 
The first hypothesis was tested by means of ANOVA. School A was significantly more 'connected' overall than schools $B$ and $D(M=3.7725$ versus 3.6462 and $3.5871 ; F=7.865=p<.001)$. When the hypothesis was tested for the subscales separately, there were no statistically significant differences between the students of the four schools regarding their connectedness to parents, siblings, peers, and the future. However, the results revealed several other significant differences. Regarding the ecological level of connectedness to others, Scheffe's post hoc test showed the following:

- Adolescents from school A were significantly more connected to their mothers than adolescents from schools $B$ and $D(M=4.2744$ versus 4.0274 and $3.9137 ; F=6.497=p<0.01)$;

- Adolescents from school A were significantly more connected to their fathers than adolescents from schools $B$ and $D(M=3.9096$ versus 3.5546 and $3.4484 ; F=8.436=p<0.01)$; adolescents from school $C$ were also more connected to their fathers than adolescents from school $D(M=3.9980$ and $3.4484 ; F=8.436=p<0.05)$.

- School A adolescents were significantly more connected to friends than school B and D adolescents $(\mathrm{M}=3.7029$ versus 3.3692 and $3.3996 ; \mathrm{F}=11.312=p<0.001)$;

- School A adolescents were significantly more connected to their teachers than school B and D adolescents $(\mathrm{M}=4.0144$ versus 3.7015 and 3.5810; $F=12.294=p<0.001$ );

- Adolescents of schools A, B and D were significantly more connected to kids from other cultures than students of school $\mathrm{C}(\mathrm{M}=3.7709,3.9107$ and 4.0546 versus 3.2417; $\mathrm{F}=8.075 ; p<0.05$ and $p<0.01$ and $p<0.001$ respectively;

- Adolescents from school $B$ were significantly more connected to romantic partners than adolescents from school C (2.8826 and 2.3113; $F=3.71=p<0.05)$;

Regarding their connectedness to communities:

- School A adolescents were significantly more connected to their school than school $C$ and $D$ adolescents $(M=$ 3.9201 versus 3.5859 and $3.6507 ; F=7.237=p<0.05$ and $p<0.01$ respectively);

- School A adolescents were significantly more connected to their neigbourhoods than school $D$ adolescents $(M=2.9376$ and 2.6363; $F=3.251=p<0.05)$;

- School $A$ and $D$ adolescents were significantly more connected to reading that the adolescents of school $B$ $(\mathrm{M}=3.3257$ and 3.2572 versus $2.9522 ; \mathrm{F}=6.13=\mathrm{p}=<0.05$ and 0.01 respectively);

- Adolescents from school $\mathrm{C}$ were significantly more connected to religion than adolescents from the other three schools ( $M=4.8583$ versus 4.2991, 3.9996 and 3.9982; $F=13.883=p<0.05)$; adolescents from school A were also significantly more connected to religion than students from schools $B$ and $D$;

Figure 2 shows the means for students of Grades 8 and 11.

Figure 2: Means of Grade 8 and Grade 11

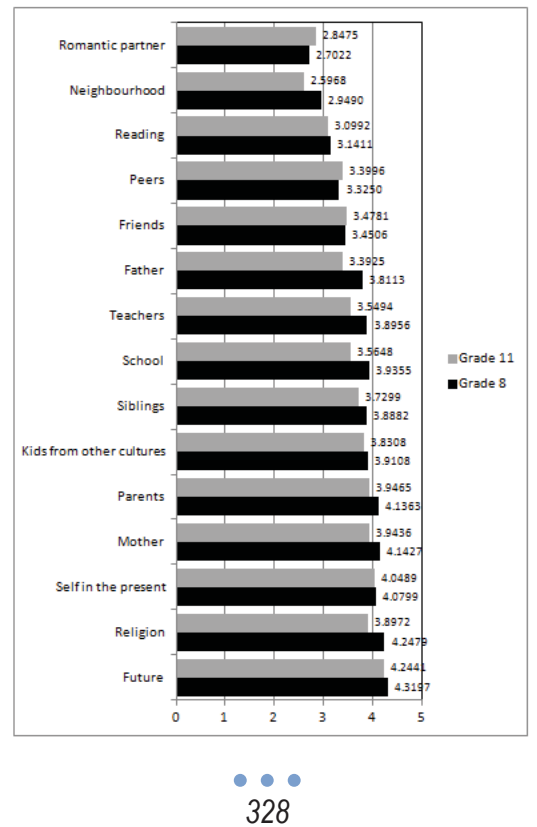


Figure 2 reveals the following regarding the two grades:

- Students of both grades were most connected to the future and to self-in-the-present $(M>4)$;

- In addition to the above, Grade 8 students were also highly connected to religion, and to their parents, particularly to their mothers $(M>4)$;

- Students of both grades were least connected to their neighbourhoods and to romantic partners $(p<3)$ although the older group ranked romantic partners higher than the younger group; both grade levels also ranked connectedness to reading relatively low ( $M=3.1411$ and 3.0992 for the Grade 8 and Grade 11 group respectively);

- The younger group ranked connectedness to school much higher than the older group ( $M=3.9355$ versus 3.5648);

- For both grades the rank order was connectedness to mothers, then siblings and then fathers; connectedness to teachers were also higher than to fathers.

The second hypothesis was tested by means of ANOVA. Overall, the Grade 8 students were significantly more 'connected' than the Grade 11 students $(M=3.7281$ and 3.5713; $F=32.266=p<0.001)$. When ANOVAs were executed for the subscales separately, no significant differences were found between the two grade levels with regard to friends, self-in-the-present, peers, reading, kids from other cultures and romantic partners. However, there were statistically significant differences between the two groups in the following instances:

Grade 8 students were significantly more connected to their parents than Grade 11 students ( $M=4.1364$ and 3.9465; $F=14.042=p<0.001)$; their mothers $(M=4.1427$ and 3.9436; $F=11.065=p<0.01)$; their fathers $(M=3.8113$ and 3.3925; $F=30.56 p<0.001)$ and to their siblings $(M=3.8882$ and 3.7299; $F=6.54=p<0.05)$. They were also significantly more connected to their teachers $(M=3.8956$ and 3.5494; $F=43.05=p<0.001)$; to school $(M=3.9355$ and 3.5648; $F=70.852=p<0.001)$; to their neigbourhoods $(M=2.9490$ and $2.5968 ; F=25.429=p<0.001)$; to the future $(M=4.3197$ and 4.2441; $F=4.082 p<0.05$; and to religion $(M=4.2479$ and 3.8972; $F=26.92=p<0.001)$ than the older students.

\section{Discussion}

In general, the adolescents of all four schools were well connected to the future, which is in line with the afore-mentioned South African study with 1,326 adolescents (Steyn et al., 2010) but in contrast to the Rawatlal and Petersen (2012) study conducted in the KwaZulu-Natal province of South Africa. In this regard two comments are relevant: the KwaZulu-Natal study was a qualitative study from which no generalisations could be made; and this province has the highest incidence of HIV/Aids infections leading to various socio-economic problems which could contribute to aimlessness (Welz, Hosegood, Jaffar, Bätzing-Feigenbaum, Herbst \& Newell, 2007). The positive future orientation of the students is an extremely valuable finding given the high incidence of societal problems South Africans face that include poverty, unemployment and violent crime (Steyn et al., 2010). This optimism may be facilitated by a religious connectedness which was also evident in the earlier South African study (Steyn et al., 2010).

Looking at the microsystem of the school, the adolescents of the four schools were, in rank order, connected to their mothers, their siblings, and thereafter to their fathers. This is in accordance with other research results worldwide (Dwairy \& Achoui, 2010; Sinha et al., 2007). The fact that the students of School A (the school that served the highest socio-economic area) were more connected to their families, is in line with statements that family connectedness seems to be stronger in families with a high socio-economic standing and educated parents (Dwairy \& Achoui, 2010).

The adolescents also revealed relatively high connectedness to religion, particularly the students in school A and C. This connectedness is deemed positively in the light of the associated psychological benefits as already explained (Houltberg et al., 2011).

In contrast to the above, connectedness to reading ranked relatively low for all students but particularly for students of school B. A lack of interest in reading is a worldwide trend for adolescents as pointed out (Machet, 2002, p. 57). Regarding the students of school $B$, the majority of the students in this school were African language students. Their lack of connectedness to reading may be related to the fact that the available books in general do not address their specific needs. For example, the books for their age group generally do not have illustrations, are not connected to a television series that they enjoy and are not in their own language. Illiteracy in English at Grade 12 level is a reality in South Africa (Van der Walt \& Hattingh, 2007) and is bound to influence the reading habits of the students.

The lack of connectedness to neighbourhoods may be a symptom of urban living. In addition, according to South African policy, the students are not expected to attend school in their own neighbourhoods. Schools B, C and D in particular may attract students who travel relatively long distances to reach their school. 
The first hypothesis can be accepted. The adolescents of school A were significantly more connectedness to others (their mothers, fathers, friends, and teachers) than the students from schools B and D. Adolescents from school C were significantly less connected to kids from other cultures, than students from the other three schools. This can be understood in the light of the fact that school $\mathrm{C}$ is a small private Christian school with white students belonging to a particular denomination and thus is particularly culturally homogeneous. Students at school B were significantly more connected to romantic partners than students at the other three schools. This issue needs further investigation but may be explained by cultural factors which are not within the scope of this paper.

The first hypothesis can also be accepted with regard to religion. The fact that students from the Christian school were significantly more connected to religion than the other students, is understandable in light of the fact that they attend a private school with a strong Christian ethos. However, the students from school A were significantly more connected to their school than those from the Christian school or the art school. This is an interesting finding and contrary to expectations. It was expected that students from school C (the Christian school) and D (the art school) would be significantly more connected to their schools since the schools focus on particular needs and interests that with which they may identify.

Regarding the mesosystem of community, significant dependencies were also identified. The fact that students from school A were significantly more connected to their neighbourhoods may be explained by the fact that of all the schools, they most serve the students that reside in their immediate (high socio-economic) environment. The significantly higher connectedness to reading of school $A$ and $D$ students needs further investigation.

Regarding the two grade levels (Grade 8 and 11), there was a similar rank order of connectedness than identified above (e.g. relatively high connection to the future and religion; relatively low connection with neighbourhoods and romantic partners; and more connected to mothers, than to siblings and lastly, to fathers).

The second hypothesis may also be accepted since significant differences were found between the two grades. The fact that the younger students were overall significantly more connected than the older students, was not expected since theoretical assumptions indicate that although connectedness with parents may lessen on some (but not all) levels as adolescents grow older, connectedness with peers and friends increases (Smetana, 2011, p. 23). The younger adolescents were significantly more connected than the older students to their families (mothers, fathers and siblings), as well as to their schools and teachers. Although school connection is deemed positively in the light of the associated advantages (e.g. Davis-Alldritt, 2012; Nutbrown \& Clough, 2009; Sánchez et al., 2005), this finding was unexpected. The Grade 8 group (who is in their first year of secondary school in South Africa), is in a new environment which is often more formal than the primary school setting and could thus be alienating, according to Loukas et al. (2009:805). In addition, they need to adapt to being the youngest/the most junior in their new surroundings. The fact that the younger students were significantly more positive about the future and religion also needs further exploration and clarification.

\section{Conclusion}

The research aimed to explore the connectedness of South African adolescents in one city in the Gauteng province and identify significant dependencies thereof with type of school and age (grade level). To this end, 486 Grade 8 and 330 Grade 11 students of four diverse schools, which included all cultural groups, completed the Hemingway (MAC) questionnaire.

Within the limitation of using a self-report questionnaire, in particular one that was not designed for the South context per se, the study was nonetheless useful for a number of significant findings. It revealed that the students were generally 'connected', with most means of 3 and above (between 'sort of true' and 'very true'). In particular, the students revealed positive personal and religious identities that supported an optimistic future orientation. Within the microsystem of the school, they were also connected to their families, in particular to their mothers, thereafter to their siblings and finally to their fathers. Their connectedness to kids of other cultures is also a valuable finding in the light of the country's apartheid history.

Some statistically significant differences between the connectedness of the adolescents of the four schools were identified. In general, the students of school A, which served a high socio-economic area with well-educated parents, were best connected. However, school $\mathrm{C}$ could consider putting structures in place to enable more inter-cultural interaction of their students and thus improve this aspect of their development. All the schools, but in particular school B, could also attend to the issue of reading and investigate ways to stimulate this interest in students.

Regarding the two grade levels (different age groups), statistically significant differences were also identified. The fact that the adolescents became less 'connected' in general and to their schools in particular, as they grow older, needs 
further investigation to be enable schools to address the matter. Moreover, the decline in religious connectedness also needs further exploration as this connection impacts on future optimism. Steyn and others (2010) have emphasised that research on the future expectations of adolescents need to be on-going for the importance of such investigations as barometer of South African social well-being.

Schools contribute to alienation and ill-being by failing to provide support for connectedness of adolescents. Adolescents need to connect to schools, communities, others and themselves for the associated psychological benefits without compromising healthy autonomy. It is hoped that this research has made a contribution to point the way for sound adolescent development in South Africa.

\section{References}

Allen, K.A. \& Bowles, T. (2012). Belonging as a guiding principle in the education of adolescents. Australian Journal of Educational \& Developmental Psychology, 12, 108-119.

Bandura, A. (1989). Human Agency in social cognitive theory. American Psychologist, 44, 1175-1184.

Bosma, H. \& Gerslma, C. (2003). From early attachment relations to the adolescent and adult organisation of self. In J. Valsiner, \& K.J. Connolly, Handbook of developmental psychology (pp. 450-488). London: SAGE.

Bowlby, J. (1988). A secure base: Clinical applications of attachment theory. London: Routledge Publishers.

Bronfenbrenner, U. (1979). Ecological systems theory. Annals of Child Development 6, 187-251.

Bronfenbrenner, U. (1999). Environments in developmental perspective: Theoretical and operational models. In SL Friedman and TD Wachs (Eds.) Measuring environment across the life span: Emerging methods and concepts (pp. 3-28). Washington, DC: American Psychological Association Press.

Brutsaert, H \& Van Houtte, M. (2004). Gender context of schooling and levels of stress among early adolescent pupils. Education and Urban Society, 37(1), 58-73.

Buhrmester, D. (1990). Intimacy of friendship, interpersonal competence, and adjustment during preadolescence and adolescence. Child Development, 61, 1101-111.

Chirkov, V., Kim, Y., Ryan, R. \& Kaplan, U. (2003). Differentiating autonomy from individualism and independence: A self-determination theory perspective on internalization of cultural orientations and wellbeing. Journal of Personality and Social Psychology, 84(1), 97-110.

Davis-Alldritt, L. (2012). School connectedness/parent engagement: Critical factors in adolescent health and achievement. NASN School Nurse, 27, 286-287.

Duriez, B. (2003). Religiosity and conservatism revisited. Relating a new religiosity measure to the two main conservative political ideologies. Psychological Reports, 92, 533-539.

Duriez, B. (2004). A research note on the relations between religiosity and racism: The importance of the way in which religious contents are being processed. The International Journal for the Psychology of Religion, 14, 175-189.

Duru, E. (2008). The predictive analysis of adjustment difficulties from loneliness, social support, and social connectedness. Educational Sciences: Theory \& Practice, 8(3), 849-856.

Dwairy, M. \& Achoui, M. (2010). Adolescent-family connectedness: a first cross-cultural research in parenting and psychological adjustment of children. Journal of Child and Family Studies, 19, 8-15.

Gallant, K., Smale, I. \& Arai, S. (2010). Civic engagement through mandatory community service: implications of serious leisure. Journal of Leisure Research, 42(2), 181-201.

Haslam, S.A., Jetten, J., Postmes, T., \& Haslam, C. (2009). Social identity, health and well-being: An emerging agenda for applied psychology. Applied Psychology: An International Review, 58, 1-23.

Houltberg, B.J., Henry, C.S., Merten, M.J. \& Robinson, L.C. (2011). Adolescents' perceptions of family connectedness, intrinsic religiosity, and depressed mood. Journal of Family Studies 20, 111-119.

Johnson, O. Jr. (2012). A systematic review of neighborhood and institutional relationships related to education. Education and Urban Society, 44(4), 477-511.

Kagitcibasi, C. (2005). Autonomy and relatedness in cultural context: Implications of self and family. Journal of Cross-Cultural Psychology, 36, 1-20.

Karcher, M.J. (2000). The Hemmingway measure of adolescent connectedness. San Antonio: The University of Texas.

Karcher, M.J. (2005). The effects of school-based developmental mentoring and mentors' attendance on mentees' self-esteem, behaviour, and connectedness. Psychology in the Schools, 42, 65-77.

Karcher, M.J. (2009). Increases in academic connectedness and self-esteem among high school students who serve as cross-age peer mentors. Professional school Counselling, 12(4), 292-299.

Libbey, H.P. (2004). Measuring student relationships to school: Attachment, bonding, connectedness, and engagement. Journal of School health, 74(7), 274-283.

Loukas, A, Ripperger-Suhler, K.G. \& Horton, K.D. (2009). Examining temporal associations between school connectedness and early adolescent adjustment. Journal of Youth and Adolescence, 38, 804-812.

Machet, M.P. (2002). Young people's reading interests in South Africa. Musaion, XX(I), 44-72.

McMillan, J.H. \& Schumacher, S. (2010). Research in education: Evidence based inquiry. ( $7^{\text {th }}$ ed.). Boston: Pearson. 
Nichols, S.L. (2008). An exploration of students' belongingness beliefs in one middle school. The Journal of Experimental Education, 76(2), 145-169.

Nutbrown, C. \& Clough, P. (2009). Citizenship and inclusion in early years: understanding and responding to children's perspectives on 'belonging.' International Journal of Early Years Education, 17(3), 191-206.

Portwood, S.G., Ayers, P.M., Kinnison, K.E., Waris, R.G. \& Wise, D.L. (2005). Youth friends: Outcomes from a school-based mentoring program. The Journal of Primary Prevention, 26(2), 129-145.

Rawatlal, K.V. \& Petersen, I. (2012). Factors impeding school connectedness: a case study. South African Journal of Psychology, 42(3), 346-357.

Rude, S.S. \& Burham, B.L. (1995). Connectedness and neediness: Factors of the DEQ and SAS dependency scales. Cognitive Therapy \& Research, 19, 323-340.

Ryan, R.M. \& Deci, E.L. (2000). Self-determination theory and the facilitation of intrinsic motivation, social development, and well-being. American Psychologist, 55(1), 68-78.

Sameroff, A.J. (2000). Ecological perspectives on developmental risk. In J.D. Osofksky \& H.E. Fistzgerald (Eds.), WAIMH handbook of infant mental health groups at risk (pp. 1-33). New York: Wiley.

Sánchez, B., Colón, Y. \& Esparza, P. (2005). The role of sense of school belonging and gender in academic adjustment of Latino Adolescents. Journal of Youth and Adolescents, 34(6), 619-628.

Seiffge-Krenke, I. \& Pakalniskiene, V. (2011). Who shapes whom in the family: reciprocal links between autonomy support in the family and parents' and adolescents' coping behaviors. Journal of Youth Adolescence, 40, 983-995.

Shochet, I.M., Dadds, M.R., Ham, D. \& Montague, R. (2006). School connectedness is an underemphasized parameter in adolescent mental health: Results of a community prediction study. Journal of Clinical Child and Adolescent Psychology 35(2), 170-179.

Sharma, A. \& Malhotra, D. (2010). Social-psychological correlates of happiness in adolescents. European Journal of Social Sciences, 12(4), 651-661.

Shulman, S \& Klein, M.M. (1993). Distinctive role of the father in adolescent separation-individuation. In S. Shulman \& W.A. Collins (Eds.), Father-adolescent relationships (pp. 41-57). San Francisco: Jossey-Bass.

Sim, T.N. (2003). The father-adolescent relationship in the context of the mother-adolescent relationship: Exploring moderating linkages in a late-adolescent sample in Singapore. Journal of Adolescent Research, 18, 383-404.

Sinha, J.W., Cnaan, R.A. \& Gelles, R.J. (2007). Adolescent risk behaviors and religion: Findings from a national study. Journal of Adolescence, 30, 231-249.

Smetana, J.G. (2011). Adolescents, families and social development. How teens construct their worlds. West UK: Wiley-Blackwell.

Steyn, M., Badenhorst, J., \& Kamper, G.D. (2010). Our voice counts: adolescents' view on their future in South Africa. South African Journal of Education, 30, 169-188.

Townsend, K.C. \& McWhirter, B.T. (2005). Connectedness: a review of the literature with implications for counselling, assessment, and research. Journal of Counseling \& Development, 83, 191-201.

Van der Walt, J.L. \& Hattingh, K. (2007). Fluency and accuracy in the writing of grade 12 ESL learners. Per Linguam: a Journal of Language Learning, 23(2), 15-28.

Welz, T., Hosegood, V., Jaffar, S., Bätzing-Feigenbaum, J., Herbst, K. \& Newell, M. (2007). Continued very high prevalence of HIV infection in rural KwaZulu-Natal, South Africa: a population based longitudinal study. AIDS, 21(11), 1467-1472.

Yeung, R. \& Leudbeater, B. (2010). Adults make a difference: The protective effects of parent and teacher emotional support on emotional and behavioural problems of peer victimization. Journal of Community Psychology, 38(1), 80-98.

$\mathrm{Yu}$, J.J. (2011). Reciprocal associations between connectedness and autonomy among Korean adolescents: Compatible or antithetical? Journal of Marriage and the Family, 73(4), 692-703. 